[0212-7199 (2007) 24: 4; pp 157-159] ANALES DE MEDICINA INTERNA Copyright $\odot 2007$ ARAN EDICIONES, S.L

AN. MED. INTERNA (Madrid) Vol. 24, N. ${ }^{\circ} 4$, pp. 157-159, 2007

\title{
Alteraciones metabólicas, VIH y enfermedad vascular
}

Betancor León P. Alteraciones metabólicas, VIH y enfermedad vascular. An Med Interna (Madrid) 2007; 24: 157-159.

La aterosclerosis es un proceso inflamatorio crónico y habitualmente progresivo de origen multifactorial. Entre los numerosos factores de riesgo cardiovascular que participan de una u otra forma en el inicio, progresión y/o complicaciones de la aterosclerosis se ha señalado a los estados inflamatorios, sean o no de de origen infeccioso.

Datos epidemiológicos apoyan la existencia de un aumento del riesgo cardiovascular en infecciones crónicas. Tal asociación está descrita no solo en las infecciones causadas por microorganismos capaces de colonizar directamente la pared vascular como Chlamydia penumonie, sino también en infecciones crónicas localizadas, con escasa repercusión sistémica como la periodontitis o la infección por Helycobacter pylori.

De igual forma, parece existir mayor riesgo cardiovascular en otras situaciones inflamatorias presuntamente de origen no infeccioso, como la psoriasis o la artritis reumatoide. Además, múltiples estudios demuestran que en las alteraciones metabólicas que cursan con aumento del riesgo vascular, como el síndrome metabólico o la diabetes, existe aumento significativo de diversos marcadores de inflamación.

El nexo común que parece explicar el aumento del riesgo cardiovascular en situaciones tan heterogéneas es la existencia de un estado inflamatorio crónico de bajo grado con liberación de múltiples mediadores con acciones diversas y redundantes, no del todo conocidas, que producen disfunción y/o daño endotelial, situación protrombótica y alteraciones metabólicas proaterogénicas. Estos efectos que ocurren en la inflamación, independientemente de la causa que la inicia, se conoce como respuesta de fase aguda.

La respuesta de fase aguda produce cambios cuantitativos y cualitativos de los lípidos y lipoproteínas del plasma, revisados recientemente por Khovidhunkit (1), de perfil más aterogénico y en gran medida similares al patrón descrito como característico del síndrome metabólico y la diabetes tipo 2: disminución de colesterol-HDL, aumento de triglicéridos, con fenotipo de patrón B (LDL pequeñas y densas). La diferencia con estas situaciones metabólicas es la disminución de colesterol-LDL.

En este número se publica un estudio de Begoña Baza (2) en el que compara los cambios en el perfil lipídico de pacientes homosexuales con y sin infección por el VIH. Es un traba- jo de referencia por la homogeneidad de la población de estudio y la acertada elección del grupo control, por el estadio precoz de la infección que disminuye el posible efecto de las situaciones que surgen a medida que progresa la inmunodeficiencia y por la ausencia de tratamiento antirretroviral que influye, de diferente forma, en el perfil lipídico.

Los resultados confirman los hallazgos de otros estudios $(3,4)$ : disminución constante de colesterol-HDL y apo A1, con menor reducción de LDL y aumento de triglicéridos. Estos cambios aumentan, obviamente, los cocientes aterogénicos. La principal diferencia con estudios previos es el menor aumento de triglicéridos, la anomalía lipídica más conocida de la infección por VIH, lo que probablemente se explica por estado inicial de la infección en los casos estudiados. Cuando esta avanza la concentración de triglicéridos aumenta y disminuye el colesterol-LDL.

El mecanismo de la dislipemia parece estar relacionado con el estado inflamatorio inducido y mantenido por la infección VIH. Si estos cambios cuantitativos de los lípidos plasmáticos, con las respectivas modificaciones cualitativas y funcionales de C-HDL propias de la respuesta de fase aguda, conllevan un mayor riesgo cardiovascular es difícil de asegurar. En cualquier caso, persiste la controversia sobre la existencia de mayor incidencia de eventos cardiovasculares en la infección VIH no tratada, en la que, además de la posible participación de la dislipemia, está demostrado que el virus puede afectar directamente a las arterias coronarias y pequeños vasos cerebrales desencadenando una reacción inflamatoria con disfunción endotelial. Un estudio reciente (5) demuestra la existencia de disfunción endotelial en pacientes con infección VIH no tratados que mejora con el tratamiento antirretroviral, independientemente del tipo de fármacos utilizados y de los diferentes cambios en los lípidos inducidos por los distintos tratamientos. El estudio SMART (6) compara la evolución de un grupo de pacientes estables con más de 350 CD4 a los que se interrumpe el tratamiento antirretroviral con un grupo control que continúa con el tratamiento. En un seguimiento de 16 meses, el grupo que interrumpe el tratamiento presenta más enfermedades oportunistas y un inesperado aumento del riesgo vascular de $60 \%$ respecto a los controles que continúan 
el tratamiento. Sin embargo, pese a la notable elevación del riesgo observada, la incidencia de eventos coronarios no puede considerarse elevada $(0,8 \%)$.

Por otro lado, no está demostrada la utilidad de las tablas para el cálculo del riesgo coronario o vascular en la población con infección VIH no tratada. Es probable que infravaloren el riesgo, dado que el C-LDL tiende a estar normal o bajo, no contemplan la concentración de triglicéridos, suele ser una población relativamente joven y no se valoran los marcadores de inflamación o los cambios cualitativos y funcionales de lípidos y lipoproteínas. Es importante señalar que en esta serie, como en la mayoría de las publicadas, se observa que la prevalencia de tabaquismo es muy superior a la población normal, y, probablemente, constituye el principal factor de riesgo vascular a controlar en esta población.

Este número de Anales incluye también un trabajo de Sorli Redó (7) en el que se estudia la influencia del sexo en el síndrome de lipodistrofia y riesgo vascular asociado a la infección VIH que, en cierta forma, complementa el anterior de Baza. Es un estudio transversal en el que se compara la prevalencia de lipodistrofia y de los factores de riesgo tradicionales entre 511 hombres y 199 mujeres con infección VIH, estables, en diferentes estadios de evolución y tratamiento $(65 \%$ expuestos a IP) y solamente $12 \%$ de los pacientes sin tratamiento previo. Los resultados repiten los encontrados en estudios previos, sin diferencias entre sexos en la prevalencia de la lipodistrofia, pero con patrón preferente de lipoacúmulo en mujeres y de lipoatrofia en varones. La presencia de lipodistrofia se acompaña, en ambos sexos, de aumento en la frecuencia de factores de riesgo cardiovascular, pero con patrón diferente. En varones con lipodistrofia es significativamente más frecuente el aumento de colesterol, la hipertensión arterial, la hipertrigliceridemia y la disminución de C-HDL, mientras que en las mujeres con lipodistrofia se distinguían de las que no la presentaban por aumento del perímetro de cintura y de hipertrigliceridemia.

Con el tratamiento antirretroviral de gran actividad (TARGA) la evolución de la infección VIH ha cambiado muy significativamente, con notable reducción en las infecciones oportunistas y otras condiciones asociadas a la inmunodeficiencia, lo que se ha traducido en un aumento importante de la supervivencia. Sin embargo, casi desde el inicio de su utilización se describen alteraciones en la distribución de la grasa corporal y del metabolismo lipoproteico e hidrocarbonado agrupadas bajo el nombre de lipodistrofia, con patrón diferente entre sexos, como confirma el presente estudio. Las características metabólicas de la lipodistrofia, la tendencia a la agrupación de varios factores riesgo, la existencia de resistencia a la insulina y la ocasional presentación de infarto de miocardio inesperado en pacientes relativamente jóvenes presenta similitud con el síndrome metabólico, lo que hizo saltar la alarma sobre el mayor riesgo cardiovascular de los pacientes con infección VIH en tratamiento tipo TARGA $(8,9)$.

Los pacientes con infección VIH tratados con TARGA, independientemente de los diferentes efectos de los fármacos utilizados en la combinación, presentan con frecuencia aumento del colesterol total, colesterol LDL, hipertrigliceridemia, hiperglucemia, obesidad abdominal y resistencia a la insulina en comparación al pre-tratamiento, que son componentes característicos del síndrome metabólico. El aumento de la frecuencia de estos factores de riesgo tradicionales, la mayor supervivencia de los pacientes que prolonga el tiempo de exposición a los mismos y un posible efecto directo de algunos fármacos, especialmente los inhibidores de la proteasa (IP) justificarían un mayor riesgo cardiovascular. Sin embargo existe controversia (10) tanto sobre la mayor prevalencia del síndrome metabólico como de la existencia de un elevado riesgo cardiovascular en pacientes con infección VIH tratados con TARGA respecto a la población normal de igual edad, raza y sexo.

Un reciente estudio de Mondy (11) compara la prevalencia de síndrome metabólico en una población no seleccionada de pacientes con infección VIH con controles seleccionados por edad, raza, sexo y consumo de tabaco de NHANES (Nacional Health and Nutrition Examination Survey, 2000-2001). Los resultados demuestran que la prevalencia de síndrome metabólico en los pacientes con infección VIH es elevada (24\%) pero no superior que en la población normal $(26,5 \%)$ americana del NHANES o a la encontrada en estudios nacionales recientes, como el estudio Telde (12). Se observaron, sin embargo, diferencias en el patrón de presentación de los diferentes componentes individuales del síndrome metabólico. Los pacientes con VIH que presentaron síndrome metabólico tenían C-HDL más bajo, mayor hipetrigliceridemia, menor perímetro de cintura, menor índice de masa corporal y menos frecuencia de hiperglucemia que los controles de la población normal. En este estudio no se observó asociación significativa entre el tipo de TARGA y la aparición del síndrome metabólico. Hallazgos similares han sido comunicados otros (13). No está claro que criterios diagnósticos de síndrome metabólico deben ser aplicados en pacientes con infección VIH en los que los criterios más frecuentes son disminución de C-HDL e hipertrigliceridemia, pero con menor frecuencia que en la población no infectada con síndrome metabólico de hipertensión arterial, hiperglucemia y obesidad abdominal. Dada la redistribución de la grasa parece más aconsejable utilizar el cociente cintura/cadera que el perímetro de cintura como criterio de obesidad visceral, especialmente en mujeres (14).

Otra cuestión es si estas alteraciones metabólicas se acompañan de riesgo vascular elevado y si el diagnóstico de síndrome metabólico mejora el valor predictivo de la ecuación de Framingham, cuya aplicación en el cálculo del riesgo cardiovascular en los pacientes con infección VIH no está totalmente validada, al no existir ningún estudio longitudinal con seguimiento prolongado. En cualquier caso, la asociación de las alteraciones metabólicas asociadas al TARGA con un elevado riesgo vascular no está totalmente clarificada. Recientes estudios prospectivos bien diseñados comienzan a dar luz sobre el tema. El estudio SMART6 demuestra aumento del riesgo cardiovascular de $60 \%$ en los pacientes a los que se les suspende el tratamiento en relación a los que lo continúan. Recientemente se han comunicado los resultados de seguimiento a seis años del estudio prospectivo DAD (15). Demuestra la existencia de un aumento de $16 \%$ por año, que al ajustar por lípidos, hipertensión arterial y diabetes se reduce a 10\% anual para los IPs. El análisis para otros fármacos no es valorable por el escaso número de infartos de miocardio y el corto seguimiento. Otro resultado destacable del estudio es que la incidencia de infarto de miocardio en los pacientes en tratamiento con IPs durante seis años es de $0,6 \%$ anual, semejante a la observada en el estudio SMART ( $0,8 \%$ anual), que debe ser considerada solo como baja a moderada.

En conclusión la infección por VIH produce cambios metabólicos, especialmente disminución de C-HDL, desde los 
estados más precoces de la infección que junto a la disfunción endotelial atribuible al propio virus puede tener algún papel en la aterogénesis. Los cambios metabólicos aumentan claramente con el tratamiento tipo TARGA y probablemente también el riesgo cardiovascular, aunque parece que menos de lo que se sospechaba, tanto por el efecto del propio tratamiento como por los factores de riesgo tradicionales, que adquieren más importancia a medida que la población infectada envejece. La prevención cardiovascular debe realizarse, como en la población general de riesgo moderado, controlando los factores de riesgo y muy especialmente el consumo de tabaco.

\section{P. BENTACOR LEÓN}

Hospital Universitario Dr. Negrín. Universidad de Las Palmas de Gran Canaria

\section{Bibliografía}

1. Khovidhunkit W, Kim MS, Menon RA, Shigenaga JK, Moser AH, Feingold KR, Grunfeld C. Effects of infection and inflammation on lipid and lipoprotein metabolism: mechanism and consequences to the host. J Lip Res 2004; 45: 1169-96.

2. Baza B, Pérez de Oteiza C, Carrió D, Salgueiro M, del Romerro J. Perfil lipídico en pacientes VIH (+) no tratados. Infección VIH: ¿factor de riesgo vascular? An Med Interna (Madrid) 2007; 24: 160-167.

3. Grunfeld, Pang M, Doerrle W, Shugenaba JK, Jensen P, Feingold KR. Lipids, lipoproteins, triglyceride clearance and cytokines in human inmmunodeficiency virus infection and the acquired inmmunodeficiency síndrome. J Clin Endocrinol Metanol 1992;74: 1045-52.

4. Sángrele R, Sardetti M, Gallati H, Reibnegger G, Waachter H, Duch D. Decreased plasma concentrations of HDL Cholesterol in HIV-infected individuals are associated with inmmuneactivation. J Acquir Inmune Defic Syndr 1994; 7: 11449-52.

5. Stein JH, Cotter BR, Parker RA et als. Antirretroviral therapy improves endotelial function in individuals with human inmmunodeficiency virus infection: a prospective randomized multicenter trial (Adult AIDS Clinical Trials Group Study A 5152s). Circulation 2005; 112:II-237. abstract

6. The Strategies for Management of Antirretroviral Therapy (SMART) Study Group. CD4+ count-guided interruption of antiretroviral treatment. N Engl J Med 2006; 355: 2283-96.

7. Sorli-Redó ML, Knobel H, Montero M, Jericó C, Guelar A, Pedro-Botet J. Impacto del sexo em el síndrome de lipodistrofia em pacientes com infección por el VIH y su asociación con factores de riesgo cardiovascular. An Med Interna (Madrid) 2007; 24: 168-172.

8. Passalaris JS, Sepkowitz KA, Glesby MJ. Coronary artery disease and

human inmmunodeficiency virus infection. Clin Infect Dis 2000; 31: 787-97.

9. Carr A, Samaras K, Burton S, Law M, Freund J, Chisholm DJ, Cooper DA. A syndrome of peripheral lipodystrophy, hyperlipidaemia and insulin resistance in patients receiving HIV protease inhibitors. AIDS 1998; $12: 51-58$

10. Stein JH. Cardiovascular risk in patients with human inmmunodeficiency virus infection: incomplete data. J Am Coll Cardiol 2006; 47: 1124-25.

11. Mondy K, Overton ET, Grubb J, Tong S, Seyfried W, Powderly W, Yarasheski K. Metabolic syndrome in HIV-infected patients from an urban, Midwestern US outpatients population. Clin Infect Dis 2007; 44: 726-34.

12. Boronat M, Chirino R, Varillas VF, Saavedra P, Marrero P, Fábregas M, Novoa FJ. Prevalence of the metabolic syndrome in the island of Gran Canaria: comparison of three major diagnosis proposal. Diabetes Med 2006; 23: 1448-55.

13. Jacobson D, Tang A, Spiegelman D, Thomas AM, Skinner S, Gorbach $\mathrm{SL}$, Wanke C. Incidence of metabolic syndrome in a cohort of HIVinfected adults and prevalence relative to the US population (National Health and Nutrition Examination Survey). J Acquir Inmmune Defic Syndr 2006; 43: 458-66.

14. Jones C, Wilson I, Greenberg A, Shevitz A, Knox TA, Gorbach SL, et al. Insulin resistance in HIV-infected men and women in the nutrition for healthy living cohort. J. Acquir Inmmune Defic Syndr 2005; 40: 2002-11

15. The DAD Study Group. Class of antiretroviral drugs and the risk of myocardial infarction. N Engl J Med 2007; 356: 1723-35. 